Sociologie et sociétés

SOCIOLOGIE

ETSOCIÉTÉS

Par-delà le Blanc et la mal

Rapports identitaires et colonialisme au pays des Inuit

Beyond the White and the Evil

Identity and Relationships and Colonialism in the Land of the

Inuit

Jean-Jacques SIMARD

Volume 15, numéro 2, octobre 1983

Enjeux ethniques : Production de nouveaux rapports sociaux

URI : https://id.erudit.org/iderudit/001707ar

DOI : https://doi.org/10.7202/001707ar

Aller au sommaire du numéro

Éditeur(s)

Les Presses de l'Université de Montréal

ISSN

0038-030X (imprimé)

1492-1375 (numérique)

Découvrir la revue

Citer cet article

SIMARD, J.-J. (1983). Par-delà le Blanc et la mal : rapports identitaires et colonialisme au pays des Inuit. Sociologie et sociétés, 15(2), 55-72.

https://doi.org/10.7202/001707ar
Résumé de l'article

Le discours critique sur le colonialisme intérieur canadien dénonce couramment l'oppression qu'exercé le Blanc sur l'Autochtone. Prenant les Inuit pour cas d'espèce, on essaie de faire voir ce que dissimule cette simplification manichéiste. Que l'altérité du Blanc est une des composantes de l'identité inuite, et que ce rapport identitaire emprunte à un même champ de signification. Que la réification idéologique de l'Inuicité et du fardeau de l'Homme blanc répond à l'impératif d'expropriation des premiers habitants et justifie le ghetto technobureaucratique où ils se trouvent aujourd'hui enfermés, en marge de l'espace et de l'histoire. 


\section{Par-delà le Blanc et le mal}

\section{Rapports identitaires et colonialisme au pays des Inuit ${ }^{1}$}

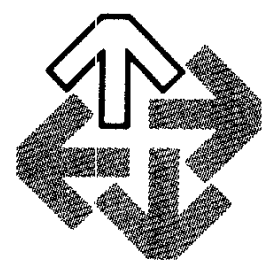

JEAN-JACQUES SIMARD

\section{I - QUAND LE BLANC PEUT ÊTRE NOIR}

Les Inuit, frappés par «les gros sourcils» des premiers Occidentaux qu'ils connurent, les baptisèrent QALLUNAAT et le terme continue aujourd'hui de s'appliquer prioritairement aux gens de langue anglaise. Cependant, selon le dictionnaire Schneider (dialecte de l'Est), QALLUNAAT désigne aussi bien l'ensemble des Blancs et même les Noirs - tout dépend du contexte, et du contexte social encore plus que sémantique.

Au sens large, en effet, les Blancs - QALLUNAAT - sont les Autres, les Étrangers, ceux qui n'ont rien de commun avec les Inuit. Il s'agit toutefois d'une altérité particulière : si un Français (OUI-OUI) ou un Noir peuvent être QALLUNAAT, les

Note: Beaucoup d'avancés de cet article s'appuient sur des sources exploitées dans ma thèse de doctorat, la Révolution congelée. Coopération et développement au Nouveau-Québec inuit (Université Laval, 1983). Si on m'attaque, je frappe avec cette brique de 750 pages, reliée plein cuir. Par ailleurs, je terminais à peine quand $\mathrm{j}$ 'ai pris connaissance d'un ouvrage de toute première qualité qui apporte beaucoup d'eau empirique au moulin que j'ai ici essayé de faire tourner. Il s'agit de la thèse en psychologie de Dominique Collin, Crise d'identité ou identité de crise? Conscience sociale et projet existentiel chez quelques jeunes Inuit du NouveauQuébec (Université de Montréal, 1983). Il y est question de choses comme de "l'image du véritable esquimau qui permet de générer [...] le groupe des Qallunaat» et d'un "ghetto de l'identité où le soi ne peut, à moins de nier son identité culturelle et ses valeurs traditionnelles, échapper à la folklorisation de son identité». Et Collin conclut là-dessus : "Il ne semble pas utile pour expliquer ce phénomène de recourir à un modèle de crise d'identité [...]. Il semble plus utile d'insister sur le rapport intergroupe asymétrique et son impact défavorable sur la construction de l'identité.” (p. 168) Le travail, qui se lit fort bien, s'appuie sur des entrevues en' profondeur réalisées auprès de jeunes adultes inuit et montagnais.

1. Le titre compte parmi les contributions diffuses apportées à cet article par Gérard Duhaime, doctorant de fond et nordiste déjà chevronné. 
Indiens forment une catégorie à part (ALLAAT). Le rapport d'identité-altérité désigné par le couple INUIT/QALLUNAAT possède en propre une charge significatrice distincte du rapport INUIT/ALLAAT. Autrefois, le mot INUK embrassait tous les véritables êtres humains (au sens du reproche adressé à l'enfant pleurnichard: fais un «Homme», fais un INUK de toi, voyons!) Les voisins - amérindiens en l'occurrence étaient déjà un peu étranges, un peu effrayants, comme le Berbère est déformé en «barbare» et que la «barbarie» porte mal visage humain. Chez les Inuit du Groënland, coupés de tout contact avec les Indiens depuis des siècles, le mot d'ALLAAT réveillait quelques esprits maléfiques et suffisait à faire trembler les pleutres (Freuchen, 1966) ${ }^{2}$. Une fois admis que les INUIT seuls appartenaient à la véritable humanité, les solidarités les plus lourdes procédaient de la parenté ou des réseaux de relations interpersonnelles (ILA), de l'identification à un headman (souvent primogéniteur) et à une aire régionale ponctuée par un camp d'hiver (formé de bandes apparentées). Autrement dit, l'utilisation du terme d'INUIT pour embrasser un groupe ethno-linguistique singulier dans le concert des cultures humaines, cet usage-là est nouveau et n'a surgi que devant le Blanc. Les significations affectées à cette conscience d'Inuicité ont été modelées au gré des rapports sociaux entretenus avec les Blancs, de sorte que l'identité de l'un renvoie immédiatement à l'altérité de l'autre comme dans un jeu de miroirs au tain fait sur mesure. Il n'y a pas d'INUIT sans QALLUNAAT et l'altérité du Blanc est une des composantes de l'identité inuit: ce sont les deux polarités d'un champ commun de signification, où l'une et l'autre se donnent mutuellement en autrui-privilégié. On n'a pas seulement affaire à une juxtaposition de différences mais à un rapport nécessaire, dialectique, structurellement ordonné et hiérarchisé. Selon un entendement qui ne se comprend pas en dehors du contexte historique où ils se sont trouvés associés, les Inuit et les Blancs sont irrémédiablement imbriqués dans un même ensemble social, ils s'appartiennent, pour ainsi dire, en propre: ils sont accouplés.

Saisir quelque chose de cette liaison oblige donc à comprendre l'évolution des structures sociales qui l'ont médiatisée, qualifiée, canalisée - ce qui se conçoit aisément. Mais alors, il faudra également convenir d'avance que l'identité inuit, toujours définie dans son rapport à l'altérité blanche, n'est pas une réalité fixée une fois pour toutes, ni ne s'attache à des traits «objectifs" empiriquement observables (chasse et pêche, chiens et iglous, primauté des rapports parentaux, et autres traditions «menacées» ou susceptibles d'être «perdues»), mais constitue au contraire un projet mouvant dans une situation historique changeante, et résulte d'une praxis symbolique où se conjuguent dialectiquement, dans le cadre restrictif d'un certain ordre social, les représentations attachées aux deux pôles de la relation identitaire - celui du Blanc aussi bien que celui de l'Inuk. Répétons-le encore autrement : on ne peut pas dire ce que c'est qu'être Inuit sans dire aussi ce que c'est qu'être Blanc, en temps et lieux donnés, et cela suppose une définition, ne fut-ce qu'implicite, du genre de relation que l'histoire et la société ont tissé entre ces deux entités. Enfin, parce que l'histoire est dynamique, toute représentation des rapports identitaires enferme aussi l'avenir, définit les possibles, constitue une option normative et idéologique prise sur l'histoire à faire, celle des Inuit comme celle des Blancs.

\section{II - LE RETOUR DES FILS DU CHIEN}

Au moment du contact - avant que ne s'instaure une relation socialement déterminée entre les uns et les autres - les Inuit n'ont reconnu dans le Blanc qu'un simple étranger auquel, en vertu d'un chauvinisme serein, ils se trouvaient supérieurs. L'explorateur Stefansonn rapporte l'amusante anecdote d'un groupe du delta du fleuve MacKenzie assez peu impressionné, en comparaison avec la magie de ses chamanes, par des lunettes d'approche qui ne permettaient pas de voir les caribous du lendemain, et par un fusil qui ne pouvait abattre le caribou caché derrière la colline (mais dont le tonitruant

2. Freuchen, P., The Book of the Eskimos, New York, Dell, 1966. 
coup de feu, en revanche, ne manquait pas d'intérêt). Une étude de Legros (1970) ${ }^{3}$ sur la même région montre comment les premiers produits manufacturés obtenus de la traite assumèrent un statut prestigieux dans les communautés inuit, mais selon les registres de valeurs et d'usage traditionnels.

Cependant, avec l'instauration d'un système mercantile fondé sur des avances de crédit qui attachaient les trappeurs au marchand de fourrures et à la production en vue de l'échange, la culture traditionnelle dut céder au marché (mondial, en l'occurrence) l'assignation de la valeur des choses. Le prix, plutôt que les hiérarchies symboliques ou utilitaires propres aux communautés inuites, devint alors déterminant. Cette distorsion est insidieuse puisque les prix, en reflétant ne fut-ce que partiellement la demande des pays riches, imposent aux Inuit une échelle de besoin extro-déterminée, qui paraît «naturelle» puisque établie par le rouage culturellement aveugle du marché. Sous le règne de la traite, la différence du Blanc ne se révèle plus tant dans le regard singulier de l'Inuk que sous la forme de rôles ou de positions sociales: le marchand, le missionnaire, le policier. Et du même trait, l'identité inuite se moule sur une série de rôles subalternes: trappeur, cathéchumène, sujet d'un Roi aussi lointain que sa Royale Police Montée du Nord-Ouest est proche. Cette condition en est une de classe, évidemment, mais l'assymétrie est vécue sous un mode personnaliste et paternaliste : c'est, selon les apparences, en vertu de qualités liées à la nature même du Blanc on dirait aujourd'hui : culture - qu'il s'occupe des Inuit comme un père de ses enfants. À s'en fier à Hugh Brody (1975) ${ }^{4}$, ces derniers éprouveraient d'ailleurs à son endroit un sentiment mélangé de crainte et de respect - le filialisme répondant au paternalisme.

Ainsi qu'en témoigne un mythe de la région d'Igloolik, les Inuit se rendent compte d'être désormais associés au Blanc et ils trouvent à cet accouplement quelque chose de monstrueux. Les Blancs seraient les descendants de la fornication maudite d'une femme inuit avec un chien. Exilés depuis les origines, ils sont finalement rentrés au pays comme la tradition l'avait prédit (Saladin, 1978) ${ }^{5}$. Le mythe, en somme, reprend à sa manière ce que nous essayons d'expliquer dans le langage de notre temps : la relation entre Blancs et Inuit est désormais nécessaire (accouplement), et en conséquence, leur identité/altérité se définit à l'intérieur d'un même champ de signification (les fils du chien et les fils de l'homme ont tous deux pour mères des femmes inuit).

Le retour des fils du chien dévoile, sous la crainte et le respect, un ressentiment et un mépris larvés, quoique résignés. Au milieu des années cinquante, dans la région des îles Belchers, le désespoir de l'assujettissement a débouché sur un autre mythe: un individu s'est mis à annoncer l'imminence d'une libération inuit conduite par le Christ revenu sur terre; à la manière du Baptiste, le malheureux entraîna même à sa suite une partie du village dans un baptême glacial en pleine Baie d'Hudson. Il y eut finalement mort d'homme avant que la Police Montée n'intervienne. Soulignons, dans cette dramatique démesure, un élément fort significatif : malgré le poids du Blanc dans la détresse des Inuit, le mythe emprunte quand même à l'eschatologie occidentale le Sauveur attendu. L'Inuk transcendera son être meurtri dans le Christ - symbole suprême du Blanc-père - ou bien il disparaîtra.

\section{III - LA MODERNISATION DE L'ÉTREINTE MAUDITE}

Après la Deuxième Guerre mondiale, le visage du Blanc change. Les ressources du sous-sol nordique excitent les convoitises du grand capital, et la maturité impérialiste cles États-Unis incite le Canada à prendre des mesures pour garantir ses prétentions géopolitiques à l'endroit du désert arctique aux frontières trop floues. Le Sauveur

3. Legros, D., Recherche en anthropologie économique: le cas des sociétés cynégétiques, l'exemple des Eskimos nunamiut chasseurs de caribou, Paris, Université de Paris X, thèse de maîtrise en ethnologie, 1970.

4. Brody, H., The People's Land: Eskimos and Whites in the Eastern Arctic, Toronto, Penguin, 1975.

5. Saladin, B., "L'homme (angut), le fils (irniq) et la lumière (qau), ou le cercle du pouvoir masculin chez les Inuit de l'Arctique central», Anthropologica, N.S., vol. XX, n 1-2, 1978, pp. 101-144. 
qu'attendaient les «nativistes» exaltés des Belchers était à leurs portes mêmes : ce serait la puissance bien temporelle de l'État. Le processus de sédentarisation atteint son terme à la fin des années cinquante, alors que le Département des affaires du Nord prend en charge l'administration des nouveaux villages et l'encadrement de la population autochtone. Une génération nouvelle et progressiste de Northern Service Officers, de pédagogues, de thérapeutes et d'agents de développement déclasse complètement les anciens portefaix du fardeau de l'Homme blanc (missionnaire, traiteur, policier). Il ne s'agit plus de «convertir», de «civiliser», de «policer», ni même de «secourir» les Inuit, mais plutôt de les «instruire» systématiquement, de les «développer», de les «organiser», de leur «fournir les services auxquels ils ont droit» - en un mot : de «les aider à s'aider eux-mêmes».

Le paternalisme personnalisé qui colorait les relations entre les anciens Blancs et les anciens Inuit sera investi dans les architectures bureaucratiques elles-mêmes. Il passera par les fonctions formelles et impersonnelles, les catégories d'expertise, les organigrammes et les matrices de programmes. Décomptés - chacun recevra son «numéro de disque» - les Inuit sont constitués en clientèle spécifique d'une branche de l'administration fédérale, et le Blanc prendra l'aspect d'un personnel spécialisé. Le profil statistique du standard de vie canadien moyen, en matière de santé, de revenu, d'éducation, etc., fournit un horizon à rattraper. Soutenues par les investissements, les dépenses courantes et les paiements de transfert du gouvernement, les marées de la consommation s'engouffrent dans le mince chenail ouvert par les marchandises de traite.

"Avant», disent les Inuit, «nous avions faim; aujourd'hui, nous n'avons plus faim». Mais ils disent aussi : "Avant, nous étions de vrais Inuit et nous nous tirions d'affaire tout seuls; maintenant nous sommes comme égarés et nous dépendons du Blanc». C'est l'idéologie officielle, reprise aussi bien dans un vocabulaire rationaliste, scientifique, critique : aguichés par les désirs du ventre et les félicités offertes par le Blanc, les Inuit ont été réduits à la dépendance; écrasés par les oppressantes attentions de la culture dominante, ils subissent les transformations que le Blanc leur impose tandis que s'effrite leur propre identité.

L'angoisse de l'authenticité culturelle forme désormais le nœud primordial du rapport identitaire: à l'Inuk tiraillé entre deux mondes, donc menacé de fausseté, correspond le Blanc déchiré entre sa responsabilité immanente d'agent de changement et sa culpabilité collective d'agent de domination et de désintégration; face aux Inuit incapables de se reconnaitre entre eux, donc victimes d'une hétérogénéité autodestructrice, se trouvent les Blancs, leur individualisme, leur concurrence, leurs débats idéologiques, leurs intérêts divergents. Une image d'épinal sert de repoussoir à la corruption et à l'effritement identitaire : celle d'une communauté inuit homogène (où chacun vit, sent et pense comme tous les siens) et une culture authentique (où les manières de vivre, se sentir et de penser sont étroitement intégrées les unes aux autres). Nul ne doute de la jauge ultime de cette authenticité et de cette homogénéité : ce sont les traits observables, les particularismes empiriques qui caractérisaient autrefois le rapport des Inuit à l'existence : la chasse et la pêche; la solidité des structures de parenté; la capacité autonome de «vivre du pays»; les coutumes, habiletés et croyances traditionnelles. La liste peut s'étendre à condition de s'en tenir à un inventaire d'idiosyncrasies manifestes et cristallisées une fois pour toutes en dehors du changement historique et avant l'intrusion de l'Altérité radicale qui confronte désormais les Inuit. J'imagine qu'on pourrait parler de réification identitaire pour étiqueter cette opération idéologique.

Tout son attrait tient à ce qu'elle correspond partiellement aux faits : les cultures de chasse et de cueillette sont effectivement très homogènes (ce qui permet à l'ethnologue d'en reconstituer presque la totalité à partir de quelques informateurs et de l'observation d'un seul cycle annuel); les séquences évolutives, les ruptures, les innovations historiques dont le cumul finit par façonner une culture particulière sont renvoyées aux origines par la mémoire mythique de sorte que l'ordre des choses, à tout moment donné, entre dans l'éternité et l'immuabilité (ce ne sont pas les Inuit qui ont raconté aux ethnographes que la culture dite «de Dorset» a précédé celle qu'on dit «de Thulé» 
et que l'on associe aujourd'hui aux Esquimaux); enfin, il est exact que la dépendance place les Inuit dans une position où ils subissent passivement beaucoup d'innovations plutôt que se les assimiler créativement.

La critique de la dépendance a d'abord visé les attitudes rétrogrades, le paternalisme grossier, l'ethnocentrisme obtus, les intérêts crus des Blancs d'autrefois - et réciproquement, la résignation naïve des Inuit d'autrefois. Ainsi, vitupère le grand ethnographe Diamond Jenness sur le dos du régime de traite :

...c'était l'homme Blanc qui donnait le ton, et l'Esquimau qui dansait sur cette musique. C'était le Blanc qui décidait de la location des postes de traite, qui décidait quels produits il devait importer au Nord, quel prix il allait en demander et combien il devait payer les Esquimaux (...) pour leur travail et leurs fourrures $\left(1968\right.$, pp. 128-129) ${ }^{6}$.

Au milieu des années soixante, l'histoire de monstres typique racontait comment il fallait, dans l'ancien temps, empiler les peaux de castor aussi haut qu'un fusil pour acheter l'arme. (Le système, ajoutait-on avec humour, se retournait contre le traiteur au pays des Inuit qui, à défaut de castor, empilaient d'épaisses pelleteries de renard blanc.) On comptait sur les lumières du relativisme culturel, le désintéressement de l'État libéral, les analyses rigoureuses de l'anthropologie scientifique pour corriger les distorsions têtues de l'ancien ethnocentrisme blanc et les appétits effrénés du capitalisme sauvage. La nouvelle génération de Blancs, pragmatique, rationnelle, éclairée, saurait bien renverser le cours des choses, en collaboration intime avec les Inuit - bien sûr, ils avaient eux-mêmes «pris conscience» de leurs problèmes et n'abordaient plus la nouveauté bouche bée. D'ailleurs, la liquidation finale de toute forme de dépendance n'était qu'une question de temps; le mot d'ordre des fonctionnaires gouvernementaux le disait: «To work ourselves out of a job.» Grâce à l'éducation populaire sur le tas, les postes provisoirement occupés par des Blancs s'ouvriraient demain à des Inuit: dès lors, ils deviendraient maitres de leur destinée collective.

Dix ans après Jenness, vingt-cinq ans après la relève de la garde, pourtant, le best-seller de Hugh Brody, The People's Land. Eskimos and Whites in the Canadian Arctic, serait forcé de reprendre, contre la bureaucratie gouvernementale cette fois, le même genre de tirade:

Il n'y a littéralement aucune partie de la vie esquimaude que les Blancs aient laissé à l'abri des domaines de responsabilités sudistes. Les Esquimaux reçoivent des maisons, des écoles, des croyances, une éducation sexuelle, des vêtements et des biens qui sont tous de manufacture et de conception blanches $(1975, \mathrm{p}$. 99 . Les traductions sont nôtres) ${ }^{7}$.

L'histoire de monstre classique, à ce moment, pointait du doigt les manuels scolaires où «Jack and Jill» s'amusaient avec des petits lapins sous les épinettes, plutôt qu' «Abilie et Sala» ${ }^{8}$ avec de petits phoques sur la banquise.

\section{IV - LE FARDEAU DE L'HOMME BLANC}

Comment expliquer cette transcendance obstinée de l'oppression? Qu'est-ce qui fait donc courir le Blanc - et piétiner les Autres? De prime abord, il semble que la volonté de puissance innée des bureaucrates s'emboutit naturellement dans l'intolérance morale et le mercantilisme des anciens fantassins du colonialisme. À quoi tient cette continuité ? Nul n'oserait plus invoquer la supériorité objective de l'Occident. Si le fardeau de l'Homme blanc lui pèse toujours, c'est à l'envers de Kipling, sous le mode de la culpabilité collective et non comme un mandat de civilisateur universel hérité de la Providence ou de l'Histoire.

6. Jenness, D., Eskimo Administration, $V:$ Anulysis and Reflection, Montréal, The Arctic Institute of North America, Technical paper $\mathrm{n}^{\circ} 21,1968$.

7. Brody, H., op. cit.

8. «Abel» et «Sarah», en phonétique inuit. 
Sous l'influence heureuse du regain marxiste en anthropologie, quelques voix se sont faites entendre, pour dénicher, sous la surface des mauvaises intentions, les rouages aveugles de la division du travail et des modes de production, de l'échange inégal à l'échelle des empires et des rapports de classe. De rares inuitologues (par exemple, Saladin, 1978 et 1980$)^{9}$, ont même suivi ces pistes explicatives jusque dans la culture traditionnelle pour y trouver, au lieu de l'harmonie et de l'homogénéité, des tensions fondamentales, des inégalités catégoriques, des violences irrépressibles. Mais la plupart des études qui se penchent ainsi sur les structures sociétales et les processus historiques dissimulés par le conflit culturel ne se sont intéressées qu'à l'époque du colonialisme mercantile : l'exploitation des trappeurs autochtones se prête plus facilement à une analyse économiste classique de la domination que leur condition actuelle de clientèle entretenue à coup de millions et d'attentions étatiques de toutes sortes - pour ne rien dire du fait que le travail productif des indigènes n'est en aucune façon nécessaire au maintien de l'ordre présentement établi. En conséquence, le petit coin marxiste enfoncé dans le manichéisme culturaliste n'est pas arrivé à le déstabiliser. Au contraire, le discours entendu le plus souvent en a gommé, comme d'habitude, les nuances originales, pour dénoncer avec encore plus de virulence les malices de l'Homme blanc, désormais crédité "culturellement» de tous les méfaits du Capitalisme en personne de telle façon qu'il soit presque impertinent de se demander si c'est le Blanc qui est spontanément capitaliste, ou si c'est plutôt le Capital qui est sorti directement de la cuisse du Blanc.

Les citations précédentes - si banales qu'elles déclencheront à coup sûr les applaudissements approbatifs de n'importe laquelle audience spécialisée - en témoignent : ce ne sont, à l'âge de la traite des fourrures, ni les mécaniques du marché, de la propriété et de la concurrence, ni l'impératif du profit qui décident de la localisation des comptoirs ou du prix des marchandises. Non, c'est le Blanc. Le même qui, plus tard, conçoit et manufacture, plans et devis en main, tantôt un modèle de socialisation l'école - à Son goût, tantôt la mode vestimentaire, ici l'habitat et là les mœurs sexuelles avec mode d'emploi, etc., jusqu'à ce «qu'aucune partie de la vie esquimaude» n'échappe à l'emprise de Son intentionalité.

En somme, de la même manière que l'identité inuit demeure la même malgré (voire même : contre) les courants de l'histoire, l'identité du Blanc se maintient telle qu'en elle-même depuis les origines de l'accouplement monstrueux, c'est-à-dire le moment du Contact. À défaut d'invoquer, comme Rousseau, la nature, le concept de culture fera aussi bien l'affaire pour l'expliquer. Une fois admis, en effet, que l'être humain est façonné profondément (jusqu'au fond de l'âme ou de l'inconscient) par le milieu singulier où il apprend à vivre, sentir et agir, le résultat observable reste le même : les gens appliquent spontanément un programme culturel (ou «idéologique» dans le vocabulaire influencé par le marxisme) dont ils sont souvent aussi inconscients que s'il leur était venu des gènes. Que cette programmation résulte des propagandes d'une minorité régnante, ou qu'elle procède plutôt d'un héritage symbolique commun à toutes les classes, n'y change rien: en bout de ligne tous et chacun des Blancs s'avèrent «naturellement» prométhéens, calculateurs, dominateurs de leur environnement (humain ou physique), rationalistes, poussés au dépassement, goal-oriented, imbus d'orgueil. En revanche, les Inuit (ou les Mbolongo, ou les Tzlatctl) sont, par culture, le contraire absolu de tout cela.

Le Blanc incarne l'Universel contre la Singularité de l'autre, et cela, indépendamment des prétentions blanches à l'universalité et à l'humanisme (prétentions vertement et communément discréditées). Voyez la Blanchitude : elle efface tous les particularismes ethniques, linguistiques, religieux, régionaux. L'indigène, au contraire, est d'abord d'un pays, d'une langue, d'une tribu, d'un clan, d'un village, d'une grande famille à nuls autres pareils. Voilà comment des Français et des Japonais, des femmes

9. Saladin, B., op. cit. et la Parole changée en pierre, Québec, Éditeur officiel, ministère des Affaires culturelles, 1980. 
instruites du Lac-Saint-Jean ou des mécaniciens noirs convertis à l'Islam, risquent de se retrouver, avant tout et indistinctement, QALLUNAAT. Voilà pourquoi des Dénés (Indiens des Territoires du Nord-Ouest), vous affirmeront ne pas pouvoir former des coopératives aussi longtemps que le principe blanc «un membre/un vote» s'opposera au mode authentiquement déné d'exercice de l'autorité, la primogéniture mâle. On pense encore au droit à l'excision du clitoris, revendiqué contre le colonialisme culturel du féminisme occidental.

Le relativisme culturel, après avoir libéré la commune humanité des lois supposées inéluctables de l'évolution, finit par se transformer en idéologie identitaire pour justifier une autre sorte de cage de fer: celle de l'appartenance fatale et de la fidélité aveugle aux héritages culturels donnés, prescrits, arrêtés une fois pour toutes et imperméables à toute espèce de questionnement. Dans la blancheur et l'inuicité, il n'y a pas que les discriminations du cour qui disparaissent : elles dissolvent aussi bien, chacune de leur bord, les classes et les sexes, avec les générations et les occupations, les couleurs des rosaces idéologiques avec les solidarités de l'engagement ${ }^{10}$. Elles laissent un univers fendu en deux. Le milieu officiel des affaires indiennes, par exemple, en est depuis longtemps rendu à l'expression Indien!Non-Indien, et cet usage tend à se répandre du côté de l'Arctique, sous l'impulsion - entre autres - des mêmes pusillanimités qui, ayant censuré par grandeur d'âme le vocable «esquimau», daignent maintenant rendre la politesse en cachant la nudité du Blanc sous le manteau du «Non-Inuit».

Les teintes idéologiques du fardeau de l'Homme blanc se sont modifiées au gré des siècles mais en restant toujours à l'intérieur d'un spectre paternaliste qui s'étend de l'ultra-blanc (il faut convertir, civiliser, ou assimiler le maudit Sauvage) jusqu'à l'infrablanc (il faut protéger le bon Sauvage). Sous le régime français, par exemple, les jésuites avaient fondé quelques villages de concentration - appelés Réductions - afin de protéger la naiveté primitive des Sauvages contre l'influence alcoolique, libidineuse, dissolue et mercantile des «François», tout en les initiant à l'agriculture et aux prescriptions de la vraie foi ${ }^{11}$. Plus pessimiste, peut-être, Denonville écrivait au ministre de la Marine, responsable de la Colonie :

Monseigneur, je vous assure qu'ils nous communiquent fort tout ce qu'ils ont de plus meschant et ne prennent eux-mesmes pour ce qu'il y a de mauvais et de vitieux en nous (cité par Perrault, 1981, p. 105) ${ }^{12}$.

On pourrait de la sorte aligner les illustrations et montrer le balancement des attitudes du positif au négatif, du bon Sauvage au maudit Sauvage, selon les milieux sociaux ou les époques. Quand le Blanc, enfermé dans l'idéologie coloniale, ne retrouve pas dans le miroir de l'Autre tous les maux dont il croit devoir se guérir, il y déniche toutes les vertus qu'il croit devoir acquérir: l'ivrognerie, la promiscuité, l'indolence, l'inconstance, etc.; ou bien, à l'opposé, la sagesse innée, la joie de vivre, l'indépendance, l'harmonie avec les autres et la nature, etc. Modernisé, le lexique parlera plutôt de désagrégation sociale, de délinquance, d'éthylisme ou d'assistance sociale; à moins qu'il ne trouve l'indigène naturellement porté au socialisme, à l'écologie, ou au bonheurdans-sa-peau. Ce tiraillement peut engendrer à l'endroit du Sauvage aussi bien le mépris que la pitié, la répression ouverte que la culpabilité rentrée. D'où, bien sûr, les figures du bon Blanc et du mauvais Blanc : le jésuite contre le commerçant d'alcool; le fonctionnaire couvant ses Indiens contre le colon envahisseur; l'anthropologue sensible contre le chasseur sportif.

C'est dire que le Blanc et l'Autochtone puisent au même bassin idéologique lorsqu'il s'agit de définir les rapports identitaires. Le concert des figures accouplées qui

10. Après avoir commandé à l'auteur de ces lignes un bref article sur la situation des Indiens du Canada, une revue mexicaine d'obédience théologique de la libération a décidé de ne pas le publier, parce que la perspective retenue était «trop blanche». On n'ose s'humilier ainsi en public que par souci d'intégrité intellectuelle : que le présent lecteur se tienne pour averti.

11. Des expériences similaires furent tentées ailleurs; en Amérique du Sud et en Chine, entre autres.

12. Perreault, I., "On débarque en Nouvelle-France», Recherches amérindiennes au Québec, vol. XI, $\mathrm{n}^{\circ} 2$, 1981, p. 103-107. 
s'appellent et se répondent organise un champ sémiologique commun. Le plus souvent, ces polarités taraudent les consciences individuelles mêmes - l'écartèlement du cœur tenant lieu, en l'occurrence, d'inconfortable point milieu où se loge la vertu. De chaque côté : le gouffre où, à tout instant, le mépris-de-l'autre peut se déverser dans le méprisde-soi.

Historiquement, les idéologues coloniaux les plus actifs ont été fournis par le Blanc - les scientifiques ou experts d'aujourd'hui remplissant une fonction similaire à celle des missionnaires. Dès que le changement est identifié, par tous les acteurs concernés, aux apports de l'Homme blanc, et que, réciproquement, le Sauvage en est le matériau ou la victime involontaire, la responsabilité ultime de la définition, de l'orientation et du rythme de toute innovation revient nécessairement au Blanc. Se pose alors un problème quasiment insoluble: le souhaiterait-on qu'il n'est pas possible (à l'échelle collective sinon pour quelques individus), d'empêcher toute corruption de l'intégrité culturelle de l'Amérindien ou de l'Inuk, ni de l'émanciper entièrement de sa condition pour en faire un «chrétien», un «citoyen», ou un «Homme» comme les autres. On ne peut ni éviter, ni court-circuiter le changement. Mais si les vertus attribuées au «non-civilisé» tiennent précisément au fait qu'il ne l'est pas, comment le «civiliser» ou le «développer» (même graduellement) sans le ronger irrémédiablement? Comment, disons, l'Inuk pourrait-il conserver son essence, son identité profonde, son authenticité, quand il est entendu que tout cela réside, en dernière analyse, en arrière et en dehors de la situation coloniale où s'exercent les forces historiques de transformation?

La pratique illustre cette contradiction. Voyez les jésuites: dès le départ, ils veulent isoler les Indiens des Réductions contre les effluves ambiantes tout en les exilant de leur être «original» par la sédentarisation, le prosélytisme et la conversion à l'agriculture. Plus tard, au dix-neuvième siècle, quand a commencé la mise en valeur agroindustrielle massive du territoire canadien, le fardeau de l'Homme blanc est retombé sur les épaules du gouvernement du Canada-Uni, puis, après 1867, du fédéral. C'est alors que, dans la foulée des Réductions, la vie en "Réserve», sous la tutelle de l'État, s'étendit peu à peu à tous les groupes amérindiens (rejoignant les Inuit après la Deuxième Guerre). Tandis que la nostalgie officielle canonisait l'Indien-du-bois, autonome et nomade, elle se retournait contre elle-même pour encourager en même temps l'agriculture (encore), la scolarisation des enfants, la formation professionnelle des adultes et bien d'autres initiatives liées à la sédentarisation et à l'économie "blanche». À présent, les mêmes voix qui mystifient la vocation écologique et cynégétique des Autochtones de l'arrière-pays et brandissent sans cesse l'épouvantail de l'authenticité menacée, passent, sans même une virgule, à la rhétorique de l'accès aux instruments de pouvoir, de la prise-en-charge, de la conscientisation, de la maitrise du développement, etc. L'angoisse identitaire qui couve derrière ces incongruités laisse également des traces dans l'ordre symbolique. Par exemple, dans l'historique ambiguité que soulève le stéréotype du «truchement-apostat» de la Relation du père LeJeune ${ }^{13}$, ni chrétien ni montagnais et traitre aux deux, dont la figure n'a cessé de se reproduire dans celles du sang-mêlé, du métis, jusqu'au jeune instruit et «acculturé» contemporain. $\mathrm{Ce}$ demi-civilisé, corrompu et faux, tout intermédiaire absolument indispensable qu'il fut entre le Blanc et l'Indigène, mérite quand même les haut-le-cœur parce qu'il réveille constamment les culpabilités du fardeau de l'Homme blanc.

Ou s'agirait-il plutôt du fardeau du Sauvage : celui de la pérennité, de l'homogénéité et de l'intégrité culturelles? On sait bien qu'en réalité, les deux fardeaux n'en font qu'un, comme le démontrent, pour qui en douterait, les propos et les gestes des leaders autochtones qui, à l'heure de la participation institutionnalisée, occupent une place de plus en plus large dans les appareils (idéologiques et administratifs) qui encadrent les Indiens et les Inuit : ils disent et font, en gros, la même chose que les Blancs qu'ils ont déplacés. Le fardeau de l'Homme blanc vient avec les postes de responsabilité prescrits par l'ordre colonial, comme guirlande en Hawaï. Aussi n'appartient-il plus en propre aux Blancs. À tous points de vue, on serait malvenu de s'en plaindre.

13. Relation de 1634, Relations des Jésuites, Montréal, Éditions du Jour, 1972. 


\section{V - LA RÉDUCTION}

Notons, à propos des villages de concentration fondés par les jésuites de Nouvelle-France, un fait conjoncturel dont la signification historique s'avérera, à long terme, primordiale : ils répondent surtout aux «besoins» d'une catégorie particulière d'Amérindiens. À Laprairie, il s'agit surtout d'Iroquois convertis au catholicisme ${ }^{14}$, donc rejetés par les leurs (pour qui le Français et ses Robes noires sont l'ennemi). $\AA$ Sillery, ce sont des Hurons ayant fui leur pays ancestral des Grands-Lacs pour échapper aux Iroquois (et aux épidémies) qui achevaient de les décimer. Dans la région de Sorel, des Abénaquis chassés de Nouvelle-Angleterre par les Bostoniens (la tête de leur chef, Mentecoumet, sera fichée en pique sur la grand-place du berceau de la Révolution américaine). Bref, des groupes dépay sés, apatrides. Ce sont déjà des exilés de l'intérieur, culturellement et géographiquement. Anormal à l'époque - la contribution des chasseurs nomadisants et des guerriers est essentielle à l'emprise de traite - ce statut anticipe néanmoins de la future condition autochtone. Car l'économie agro-industrielle, au contraire, pouvait se passer des autochtones; plus encore : leur simple titre de premier habitant, leur nomadisme, leur enracinement symbolique dans un territoire ancestral entravaient la libération capitaliste de la terre, c'est-à-dire sa transformation juridique en facteur de production librement aliénable (aux conditions d'un marché parmi d'autres). Sous le nouveau mode de production, en somme, la place spécifique des Indiens, c'est de ne pas avoir de place. À moins de les exterminer à coup de fusil (pour liquider un travail sale déjà bien amorcé par les maladies contagieuses), ou de leur nier juridiquement toute spécificité (l'assimilation unilatérale), il fallait bien de quelque manière les sortir des territoires à aménager, les mettre «en réserve» de l'espace, les concentrer ponctuellement dans des établissements hors desquels ils ne seraient plus chez eux. C'est cet impératif d'expropriation, qu'on vient d'appeler «l'exil intérieur», qui forme le noyau dur du «problème autochtone» au Canada (comme dans tous les pays développés où les habitants d'origine forment une minorité : États-Unis, Australie ou Brésil, par exemple).

En certain cas, l'État a tenté de libérer le sol selon la procédure du contrat de vente, par des «traités» conclus avec quelques "nations» régionales. Outre l'écart ridicule entre les termes de l'échange, ces rituels étaient illégitimes puisqu'ils reposaient sur un malentendu entre les conceptions bourgeoises de la propriété et un droit différent, où l'idée même que la terre puisse appartenir aux humains plutôt que ceux-ci à celle-là, et qu'ils puissent l'aliéner à leur guise, équivalait à une véritable hérésie. La légitimation de l'expropriation autochtone dut donc, en pratique, passer par d'autres canaux, dont, pour l'essentiel, l'institutionnalisation du fardeau de l'Homme blanc.

Au milieu du dix-neuvième siècle, l'expansion de la colonisation et de l'industrie forestière dans l'arrière-pays canadien bouleversa l'équilibre écologique et économique de la traite des fourrures. Les Indiens ne furent pas seuls à s'en plaindre : les missionnaires et la Compagnie (monopoliste) de la Baie d'Hudson essayèrent aussi de résister. Peine perdue. Poussés par la misère, la maladie - toujours elle, cette Conquérante plus sournoise que tous les autres - le mépris des immigrants du voisinage, la concurrence des chantiers, de l'agriculture, et plus tard, des mines, de la chasse et de la pêche sportive, des parcs nationaux et provinciaux, des barrages hydro-électriques, les Indiens durent se réfugier dans les Réserves; puisque le problème concernait l'ensemble du pays et que les Provinces, constitutionnellement responsables des terres et des richesses naturelles, ne voulaient rien savoir d'eux, le gouvernement fédéral hérita de leur entretien. Il poursuivit dès le départ une politique relativement «progressiste», en ce sens que, par certains aspects, elle correspondait déjà à celle d'un État-providence plutôt que d'un État-gendarme ${ }^{15}$. On ira, dans le langage d'aujourd'hui, «au devant des

14. C'est là que mourra Sainte Kateri Tekakhouita.

15. Attention : à la moindre résistance ouverte, le Gendarme sortait la matraque que la Providence cachait derrière son dos. Les Mohawks (Iroquois) qui se sont opposés à l'abolition de leurs structures politiques l'ont appris à leurs dépens dès la fin du dix-neuvième. Encore l'an dernier, le gouvernement du Québec a 
besoins des administrés», au lieu d'attendre l'urgence et les plaintes; on favorisera la «participation» en créant des conseils de bande aux fins de consultation locale; les fonctionnaires régnant sur les réserves agiront aussi bien comme «animateurs sociaux» et «agents de conscientisation» auprès de leurs pupilles, plutôt que de se contenter du simple rôle de gérant. Inutile d'ajouter que ces tendances ont connu leur apothéose après 1950, quand se diffusa massivement d'un bord à l'autre du pays le modèle, désormais normal, de l'État interventionniste, planificateur, redistributeur, ouvert à la «démocratie de participation», bref, l'État technocratique en pleine maturité.

En conséquence, il est malaisé de parler d'exploitation, d'extorsion, de prolétarisation, ou, au sens strict d'Amin, de développement inégal, pour décrire la condition des autochtones. Je préfère pour ma part emprunter à l'histoire le concept de réduction et le souffler en paradigme d'un confinement, d'une émasculation, d'un rétrécissement qui ne concerne pas exclusivement l'espace vital des Indiens et des Inuit, mais l'horizon entier de leur vie sociale et de leurs rapports au monde. Ils ne se trouvent pas seulement mis-en-réserve de l'espace, ils le sont aussi de la production, de la praxis politique, de la responsabilité même de concevoir des possibles et des projets identitaires. Une cloche de verre sociologique les recouvre et les enferme, hors de l'espace et hors du temps. Ils sont aussi placés en réserve de l'histoire.

Les Réserves rétrécissent dramatiquement l'accès des autochtones au territoire environnant, cela tombe sous le sens. Cette réduction spatiale entraîne une réduction socio-économique. Les restrictions à la mobilité saisonnière imposées par l'école ou le dispensaire, ou par la concurrence du mode capitaliste d'appropriation des ressources, démanchent les rouages de l'économie domestique et refoulent les anciens chasseurscueilleurs dans le corridor étroit de l'artisanat, du guidage, du bûchage ou du bâtiment occasionnels, des services gouvernementaux, du petit commerce de la boutique. L'insécurité économique, le gaspillage et dans une triste mesure, la misère sont en conséquence courants en Réserve et c'est dans ce terreau quotidien que la réduction socio-politique plante ses racines.

$\mathrm{Si}$, par politique, on entend le domaine où les membre d'une société exercent une action réfléchie sur leur société même, les Autochtones sont doublement confinés sur ce front là aussi : handicapés en matière de participation à la «politie» canadienne - leurs votes, par exemple, sont noyés dans des circonscriptions électorales où ils sont presque toujours archi-minoritaires; et leur curieux statut de pupilles de l'État fédéral en fait des demi-citoyens au regard des États provinciaux - ils ne sont guère en mesure, non plus, d'agir librement sur la vie publique de leurs propres communautés. Ils n'ont que partiellement part à la souveraineté et à la responsabilité civile telles que définies, disons, par la Déclaration universelle des droits de l'Homme. En revanche, ils subissent un enveloppement administratif extraordinairement totalitaire (par rapport aux normes d'une démocratie libérale). Une fois passé le niveau des relations primaires (familles et réseaux interpersonnels), l'organisation sociale des Réserves se moule presque exclusivement sur les appareils d'État, publics ou parapublics. Les superstructures pseudo-associatives censées les associer politiquement - conseils de bande et associations ethniques ou nationales - ont de moins en moins de base politique à mesure qu'elles s'éloignent des localités immédiates et tiennent l'essentiel de leur légitimité (et de leurs moyens) du statut d'interlocuteur officiel qui leur est bureaucratiquement concédé par les organes étatiques chargés de la gestion des autochtones. Consultés à mort (politique) ils n'ont guère d'occasion de débattre, entre eux et synthétiquement, des affaires de tout le monde, d'en assumer la responsabilité et de porter les conséquences de leurs décisions collectives. Au lieu de leaders, ils se contentent d'une espèce édulcorée de fonctionnaires; à la place d'un gouvernement, de conseils sectoriels et d'administration; et pour remplacer les projets collectifs, de "programmes» et de «crédits budgétaires».

mandaté 250 agents pour aller casser les reins des Micmacs de Restigouche $(750$ h.) qui pêchaient «illégalement» le saumon. 
Bien sûr, les exceptions ne manquent pas, de coopératives, d'entreprises indépendantes, d'associations communautaires qui se tirent d'affaire en dehors des appareils d'État. Mais ce sont, précisément, encore des exceptions. Bien sûr, les instances amérindiennes ou inuit officielles revendiquent de plus en plus une forme quelconque de participation à la souveraineté. Mais, en pratique, cela ne veut rien dire encore .

La réduction idéologique vient fermer la boucle, et rendre tout le reste fatal, que dis-je? vertueux. La refoulement sur l'économie de village et les activités marginales? Il répond à la vocation auquelle l'histoire et la culture ont si bien préparé les autochtones : cynégétique et halieutique, communautaire et écologique, ne vient-elle heureusement compléter en ce monde notre vocation hydro-électrique ou minière, au moins d'envergure nationale et naturellement industrielle? L'encoconnement politique? Mais il réalise le droit à la différence, découle des caractères singuliers des clientèles autochtones, témoigne de l'attention particulière que doivent leur porter les gouvernements «pour réparer des Blancs l'irréparable outrage». Leur destin, c'est la survivance, l'endurance, la conservation, la tradition - le nôtre est dépassement, maittrise des choses, projet, critique des routines et valeurs reçues. L'Histoire, le Progrès, le Capitalisme «menacent» leur identité - ils «interpellent» la nôtre. En résumé, cette sorte d'humains-là ne peuvent s'écarter de ce que "les autres ont fait d'eux», pour parler comme Sartre. Rien ne leur servirait, à la manière d' "autres" groupes ethniques, de s'acharner à poser concrètement sur un environnement mouvant la signature sans cesse corrigée de leur originalité. Pour eux - encore du Sartre - l'essence précède l'existence. Des fois, on dirait qu'elle l'empêche.

Cette idéologie ethniste (ou culturaliste, au sens empirique et positiviste du terme) ne peut tenir que par sa faculté de justifier - bien plus qu'elle n'explique, eut ajouté Fernand Dumont - la marginalisation des premiers habitants, et, en dernière analyse, leur expropriation. D'où mon expression: «mise-en-réserve de l'histoire». Il m'est difficile de ne pas penser aussitôt au nationalisme canadien-français de l'Entre-deux-guerres. N'étions-nous pas, après tout, aux lendemains de la conquête, les «indigènes " des Anglais? La grand-ville, la science, le matérialisme, l'industrie, l'accumulation, etc., appartenaient, n'est-ce pas, à leur «race» et non à la nôtre?

Ou était-ce que nous aurions souhaité arrêter la course de l'histoire pour en débarquer? En tout cas, quand notre Grand Sachem René Lévesque a offert en hommage aux dignitaires amérindiens «le couteau du chasseur» (rapporté par Gourdeau, 1979) ${ }^{16}$, je me suis dit que ce beau geste équivalait à celui d'un MacKenzie King remettant à Maurice Duplessis le chapeau de paille de cultivateur ${ }^{17}$.

\section{VI - IDENTITÉS AUTHENTIQUES ET FAUX POUVOIRS}

Si le qualificatif d'ethniste qu'on vient d'appliquer à l'idéologie de la réduction rappelle celui de «raciste», tant mieux. Il y a quelque parenté entre les deux. Entre autres, ce qu'on pourrait baptiser «subjectivisme sociologique» : cette propension à traiter les catégories sociales comme des Grandes Personnes dotées de tous les attributs d'un sujet individuel mû par des pulsions, des intentions, une volonté qui lui sont propres, de telle manière que chaque individu susceptible d'appartenir à cette catégorie n'en soit qu'une manifestation. D'un conflit d'appropriation sociale des moyens de gagner ou de donner un sens à la vie, l'idéologie ethniste fait quelque chose comme un titanesque conflit de personnalités, de mentalités, d'attitudes collectives. L'antisémitisme, certainement, mais aussi certains romantismes anticapitalistes font de même. Cela débouche facilement sur des conceptions volontaristes, intentionnalistes, voire conspiratoires (puisqu'il faut bien que la clique s'entende) de l'ordre social: $\mathbf{M}$.

16. Gourdeau, E., «Les autochtones québécois », dans Sarrazin J. (édit.) Dossier-Québec, Paris, Stock, 1979, pp. 307-321.

17. King, d'aucuns l'ont peut-être oublié, était Premier ministre du Canada à la fin des années trente, âge d'or du nationalisme agriculturaliste canadien-français, quand commençait, du côté de Québec, le règne de Duplessis. 
le Blanc, ou M. le Juif, ou M. le Capital ou M. l'Anglais «décident de nos vies» et de tout le reste tandis que M. l'Inuk, ou M. le Gentil, ou M. le Prolétariat, ou M. le Québécois n'en finissent plus de se faire emmancher, flouer, récupérer.

Cette opération intellectuelle, pour être fautive, n'est ni sans précédent ni surprenante. Elle consiste à prendre les acteurs sociaux pour ce que nous savons tous être, par expérience immédiate de nos relations avec les autres: des individus dotés de libre arbitre. Et elle correspond, dans l'esprit sociologique, aux projections théologiques du sujet humain dans un être suprême et transcendant - les gens créent des dieux à leur image, même si leur foi affirme l'inverse - qui se porte garant de l'ordre des choses et de la vie.

Pourtant, s'il est un enseignement à tirer de l'étude et de la réflexion à ce propos, c'est bien que les catégories sociales et les acteurs collectifs sont créés par les tendances et les processus structurels régissant le fonctionnement d'un certain ordre sociétal, au moins autant que cet ordre est créé par les intentions des acteurs. D'où le mot (un peu excessif) de Marx, selon lequel «les Hommes font leur histoire mais ne savent pas qu'ils la font». L'admettre n'équivaut pas à retirer toute marge de manœuvre aux puissants - ou réciproquement, aux faibles - mais à suggérer qu'on ne comprendra rien ni à la domination ni à l'assujettissement à moins de prendre la peine de creuser sous les apparences telles que le sens les révèlent, pour saisir les conditions sociologiques qui en prescrivent l'exercice dans une société concrète, en temps et lieux donnés. Le "pouvoir», en somme, n'est pas tant une qualité inhérente aux dominants qu'une dimension des rapports sociaux, toujours organisés d'une certaine manière, selon une logique ou une dynamique que les acteurs appliquent le plus souvent sans les connaître, inspirés par la culture, l'idéologie, l'imitation ou les simples réactions adaptatives à des situations qu'ils s'efforcent de maîtriser mais dont ils ne sont pas les auteurs.

Quand on vous fait du Blanc, donc, en potentiel, de n'importe lequel blanc, un être omniprésent, omniscient (encore que privé de l'amabilité et de la bonté infinie), vous êtes en droit de flairer l'odeur du mythe et de commencer à creuser sous les évidences. Les évidences elles-mêmes, remarquez, s'y prêtent mal. Que, disons, un ouvrier puisse, dans l'espace de sa vie, devenir capitaliste n'est pas usuel, mais ça se conçoit. Par contre, il est certain qu'un Inuk défini par la singularité objective de son héritage culturel ne peut pas devenir un Blanc. L'identité devient ici un dépôt figé au fond de chaque Inuk en chair et en os, aussi indépassable que la pigmentation des cheveux, et dès lors à l'abri des circonstances et conditions sociétales.

On aura vu ce que cela suggère : remplaçons par des Inuit les Blancs qui dominent actuellement et la domination elle-même s'évanouira. Les mécanismes structurels, les formes selon lesquelles se réalise actuellement la domination n'ont aucune importance. Un propriétaire privé inuit ne saurait exploiter ses congénères puisque l'exploitation est étrangère à la culture inuit. Des fonctionnaires inuit ne pourraient davantage ratatiner les leurs dans un statut de clientèle dépendante et éparpillée en autant de secteurs que l'esprit bureaucratique peut découper dans la vie: non, dès le moment où un appareil (même déjà installé) est chapeauté par des Inuit, il cesse de relayer l'oppression étrangère et se plie aussitôt, en toute neutralité technobureaucratique, aux besoins authentiques de tous et chacun des Inuit. Qui est contre? Personne.

Personne? Alors on s'est mis à l'ouvrage à Montréal et au Nouveau-Québec, dans les officines gouvernementales et parmi les experts-consultants en affaires autochtones ${ }^{18}$, chez les Inuit comme chez les Cris, et, pressés par l'imminence du développement des ressources hydro-électriques de la Baie James, on a conclu en 1975 ce qu'il est convenu d'appeler les Accords (ou la Convention) de la Baie James.

Tels qu'ils s'appliquent aux Inuit (le régime des Cris n'est pas entièrement le même), ces accords liquident d'abord la question du titre aborigène : par euphémisme, les

18. Pour éviter tout pharisaïsme, j'avouerai avoir été du nombre. Le résultat de tous ces efforts peut être admiré dans Québec, la Convention de la Baie James et du Nord québécois, Éditeur officiel, 1976. 
Inuit y « renoncent» et reçoivent «en compensation» une soixantaine de millions. Qui ça, les Inuit? Un holding financier du nom de Société Makivik, chargé de faire fructifier les indemnités, dont sont actionnaires (de naissance ou par ratification communautaire), tous les Inuit du Nouveau-Québec, et qui, en plus du devoir de faire de l'argent avec de l'argent selon les règles usuelles de l'économie nord-américaine, s'acquitte aussi de celui de représenter officiellement les Inuit auprès des gouvernements du Sud.

Ensuite, la protection de la culture traditionnelle : territoires de chasse et pêche exclusifs, droit à l'enseignement de la langue et des traditions, comités écologiques, chasseurs-fonctionnaires pour l'approvisionnement des démunis en «nourriture du pays», etc. Et en dernier lieu, participation à part entière des Inuit à la gestion des organismes administratifs qui les «servent»: municipalités, commission scolaire, administration régionale, comités conjoints et conseils consultatifs de toute sorte. Qui est contre?

Certainement pas les représentants des Inuit. Il est entendu que tous les programmes fédéraux et provinciaux continueront de s'appliquer au Nouveau-Québec, qu'ils seront même, comme on dit, «bonifiés», et que les fonds continueront à couler de Québec et d'Ottawa. Certainement pas les représentants des Blancs : la terre est libre, et tous les pouvoirs législatifs, tous les droits de prélever impôts et taxes dans l'Inukland, toutes les responsabilités exécutives ultimes demeurent aux mains des parlements et des gouvernements québécois (provincial) et canadien (fédéral).

Même les milieux universitaires spécialisés y apporteront leur nihil obstat. Au juriste européen Norbert Rouland venu faire enquête, un ethnologue confiera que, jusqu'à la signature de la Convention on avait «eu tendance à considérer que les Autochtones avaient systématiquement été mis en état d'infériorité par la société dominante, alors que rnaintenant, ils avaient les moyens de prendre la responsabilité de leur avenir» (Rouland, 1977, p. 230) ${ }^{19}$.

Or, en réalité, l'application des accords de la Baie James a produit un renversement pervers des effets et des intentions. Le régime «nouveau» devait protéger l'économie et la culture traditionnelles, libérer les Cris et les Inuit de leur dépendance politique et économique envers l'État et ses appareils, remettre à la base autochtone grâce aux modalités multiples de "participation populaire aux prises de décision» - le pouvoir de définir et de conduire sa destinée. Selon une étude de suivi très rigoureuse, conduite en 1979 par la maison s.s.D.c.c., les résultats étaient tout autres. Les communautés locales avaient «échangé leur dépendance envers le ministère des Affaires indiennes (contre) une dépendance envers les structures régionales [...]». Celles-ci s'étaient transformées en «livreur de services» et agissaient parfois comme si elles étaient responsables «devant les niveaux supérieurs des gouvernements» plutôt que devant le peuple. «Le langage technocratique (avait) envahi la sphère de la bureaucratie régionale» et, à défaut de le maîtriser - c'est le cas de la majorité des autochtones - on ne pouvait «participer activement à ce niveau».

Sur le front écologique et traditionnel, les chasseurs-trappeurs étaient de plus en plus étroitement encadrés par des organismes de gestion dont les règlements et les ordres tendaient à remplacer ceux de la tradition. Dans le cas de deux relocalisations majeures, les avantages obtenus favorisaient «la partie de la population qui n'évolue pas dans l'économie de subsistance [...] et aux dépens de la base économique des chasseurs et pêcheurs». Ces gestes et d'autres plaçaient «les principes de conservation [...] dans une zone grise». (Toutes citations tirées de s.s.D.c.c., 1979, pp. 85, 143, $214,221^{20}$.) Les chercheurs notaient également au passage que l'essentiel des délibérations des conseils locaux ou régionaux se déroulaient en anglais.

19. Rouland, N., le Règlement du statut juridique des Inuit du Nouveau-Québec et la Convention de la Baie James et du Nord québécois du 11 novembre 1975, Université d'Aix-en-Provence, 1977. La version épurée de ce manuscrit a été publiée conjointement par le groupe Inuksiutiit et les Presses de l'Université Laval en 1979.

20. s.s.D.c.c., la Négociation d'un mode de vie. La structure administrative découlant de la Convention de la Baie James : l'expérience initiale des Cris, Montréal, 1979 (ronéo). 
L'étude, il faut le dire, portait sur les Cris, mais ses conclusions générales s'appliquent pour l'essentiel à la situation qui prévaut au pays des Inuit (voir ma thèse de 1982). Il ne faudrait pas non plus, par obsession misérabiliste, manquer d'observer que la Convention a permis aux Inuit de maintenir, malgré la crise économique, un standard de vie moyen confortable, et que nombre d'entre eux en ont tiré de solides et rémunérateurs emplois : en 1980, 205 pour les gouvernements et 376 dans les bureaux créés par la Convention (sur une population active d'environ 1800 ). Plusieurs découvrent de la sorte, et pour la première fois, comment s'exerce le pouvoir dans la société canadienne et nuls autant que les cadres supérieurs de la puissante Société Makivik (administratrice des millions d'indemnisation), qu'on reçoit avec beaucoup de politesse dans les antichambres du grand capital et les couloirs de l'Administration.

Cependant, il y avait, en 1980, 523 "Non-inuit» qui travaillaient au NouveauQuébec. Les Blancs détenaient $35 \%$ des postes salariés de la région, dont les deux tiers aux titres supérieurs d'administrateur, professionnel et technicien. Les gouvernements en employaient 205; les services parapublics, 125. Ces chiffres ne portent que sur le Nord. Ils négligent les dizaines de fonctionnaires chargés des dossiers du NouveauQuébec dans les ministères d'Ottawa et de Québec. En tout et partout, et en négligeant toute distinction technique, cette collectivité d'environ 5000 personnes mobilisait, sur le terrain même, 530 fonctionnaires publics et para-publics (Beaulieu, 1982) ${ }^{21}$.

Pour Serge Bouchard, coauteur de l'étude s.s.D.c.c. citée plus haut, la Convention constitue «une performance hautement sophistiquée de la bureaucratie étatique pour récupérer ce qui lui échappait jusque-là: le contrôle total de la destinée des populations amérindiennes» $\left(1979\right.$, p. 187) ${ }^{22}$. Je n'y souscris pas sans réserve. D'abord, la moindre expérience suggère que la bureaucratie étatique a tous les talents sauf ceux de la performance et de la haute sophistication. Le concept de récupération est inconfortable car il risque de soutenir les visions conspiratoires et volontaristes du pouvoir. L'idée de contrôle total, enfin, est hyperbolique : aucune société n'est vraiment totalitaire. Cependant, si par bureaucratie on entend un système d'intégration sociale; si par récupération on veut dire qu'un ordre social se reproduit en accommodant formellement des tensions auparavant souterraines; si on admet que l'univers des affaires autochtones au Canada forme une orbite close, une espèce «d'institution totale (selon l'entendement donné par Goffmann à ce terme); alors Bouchard a raison sur tous les points.

Seulement, cela était évident au matin même de la signature des accords, dont les effets pervers étaient parfaitement prévisibles ${ }^{23}$. La Convention n'a fait que ratifier formellement la situation qui existait au préalable. Les deux volets modernes de l'idéologie ethniste, le monolithisme identitaire et la participation de clientèle, y ont tout naturellement trouvé leur cristallisation juridique. L'insertion d'une strate de personnel autochtone entre les sommets du pouvoir colonial et le peuple n'a pas dérangé la structure d'assujettissement, ni sa logique, ni son discours. Réduite à des écarts statistiques par rapport à la norme canadienne, la condition des Inuit n'appelait que des corrections quantitatives: c'est à peu près fait. Au fronton des bureaux de négociation on eut pu inscrire le mot d'ordre: More of the same!

Aussi, même si les réalités actuelles semblent tourner le dos aux intentions avouées des signataires de l'entente, ceux-ci ne paraissent pas pressés de s'en rendre compte. Sur toutes les tribunes, par exemple, le gouvernement du Québec y réfère pour illustrer le remarquable libéralisme de sa politique autochtone. De leur côté, les représentants officiels des Cris et Inuit ont proposé, lors des conférences constitutionnelles canadiennes de l'an dernier, d'enchasser les accords de la Baie James dans la loi suprême du pays.

21. Beaulieu, D., les Coopératives inuit. Aperçu de leur milieu, Québec, ministère des Institutions financières et coopératives, 1982.

21. Bouchard, S., "Pauvres combats, tristes arènes», Recherches amérindiennes au Québec, vol. IX, $\mathrm{n}^{\circ} 3,1979$.

23. Comme en fait foi, par exemple, ma propre jérémiade, «Les souverainetés irréconciliables», Actes du congrès annuel de l'A.C.S.A.L.F., Ottawa, 1978. 
Ceci dit, les parties se retrouvent périodiquement à couteaux tirés, sur l'extension financière des obligations contractées par les gouvernements d'un côté, sur la maladministration des autochtones, de l'autre. Ajustements de parcours? Peut-être. Car il serait facile de démontrer, chiffres en main et en prenant pour barème n'importe lequel des indicateurs de la condition autochtone dans le reste du pays, que le contrat social paraphé au Nouveau-Québec demeure, malgré des imperfections à corriger, le meilleur qu'on ait actuellement.

Pourtant la plupart des organismes qui font entendre la voix des Autochtones ailleurs au pays ont tendance à considérer la Convention comme un exemple de ce qu'il ne faut pas faire. Sur le territoire même, un noyau important d'un millier de dissidents inuit répartis dans trois villages (sur treize) a refusé de la ratifier et persiste à en repousser les avantages (voir Ligue des droits et libertés, 1983) ${ }^{24}$. Ils disent que les Inuit conventionnés ont accepté l'expropriation et qu'il n'y a guère de différence entre la morsure d'un chien rouge et celle d'un chien blanc ${ }^{25}$.

Ils disent que la Convention n'a pas brisé l'accouplement monstrueux.

\section{CONCLUSION}

Nous avons essayé de comprendre comment l'opposition simpliste entre le Blanc dominateur et l'Inuit dominé embourbait, bien davantage qu'elle n'éclairait, la pensée et l'action engagées dans la lutte contre l'inertie colonialiste pesant sur beaucoup de citoyens de ce pays. Nous avons voulu montrer que la position sociale des Inuit comptait au moins autant que leur héritage culturel dans les représentations qui tentent de définir leur identité, et que celles-ci se dégageaient dialectiquement d'un rapport à l'Altérité investie dans la figure symbolique du Blanc. La même chose peut se dire autrement : tous les Inuit ne sont pas nécessairement des Inuit, et tous les Blancs ne sont pas nécessairement des Blancs à la manière prescrite par l'idéologie ethniste. Cela dépend moins de leur héritage culturel respectif que de la case qu'ils occupent dans la structure sociale d'expropriation coloniale. Ou encore: parler de société inụit et de société blanche est absurde. Les entités historiques qu'on identifie ainsi participent du même ordre social et les stéréotypes où on les enveloppe empruntent à un champ comrnun de signification ${ }^{26}$. Nous avons également tenté de faire voir comment l'idéologie dominante dans le milieu des affaires autochtones canadiennes contribuait - c'est tautologique - à la domination, en justifiant, par exemple, au nom du droit à la conservation identitaire, la réduction des Inuit au statut d'une clientèle administrative ou à celui d'une "petite bourgeoisie technobureaucratique compradore ", l'une et l'autre tout à fait agréables au colonialisme d'appareil que pratiquent aujourd'hui les États canadiens expropriateurs sur le dos des premiers habitants.

Le lecteur conclura à sa guise, cela va de soi. J'en tire pour ma part quelques hypothèses de recherche que je n'ose encore exprimer clairement à défaut de pouvoir les défendre solidement en public et d'éviter ainsi de me faire traiter - vous avez deviné - de Blanc!

Je pars de cette constatation: quand j'entends qu'on oppose les Blancs aux Inuit, cela me chicote la logique. Les premiers n'ont pas de langue en propre, ne

24. Ligue des droits et libertés, les Inuit dissidents, Montréal, en collaboration avec Inuit Tungavingat Nunamini, 1983.

25. Je n'invente pas cette métaphore. Elle a été publiquement utilisée par les porte-parole des dissidents. Voir Québec, aÉtude des projets de loi $\mathrm{n}^{i} \mathrm{25}, 27,34,42 \%$, Journal des débats, Commission permanente des Affaires intergouvernementales, troisième session, trente-et-unième législature, 21 juin 1978. Au moment de corriger les épreuves du présent article la conjoncture politique semble se déplacer. Voilà que des Inuit associés aux institutions de la Convention s'avouent déçus des résultats pratiques d'un système qu'ils avaient contribué à établir. Ils rejoignent les dissidents pour dénoncer l'aliénation du territoire, de même que l'éparpillement et le musèlement bureaucratiques de la vie et de la voix collectives des Inuit.

26. Si j'ai bien lu Alain Touraine, il nomme orientations culturelles d'une société (ou d'un système d'action historique) ce que je désigne ici par «champ commun de signification», non par souci de pseudooriginalité mais pour éviter les confusions auxquelles est soumis le terme de "culture» dans le milieu que j'étudie; d'autre part, le concept d'"orientations» (vecteurs) me paraît un peu limitatif : le champ de signification s'organise aussi bien sur une armature sémiologique profonde du type qu'étudie Lévi-Strauss lorsqu'il recompose des «structures». 
forment pas une ethnie, et n'existaient même pas avant l'ère des grandes découvertes (sauf par la pigmentation épidermique). Louis-Jacques Dorais, le linguiste de Laval, me dit que la langue «inuttitut», telle que parlée aujourd'hui, s'est écartée d'un tronc commun il y a de cela aux environs de sept milliers d'années. Cela suffit pour soupçonner qu'en comparant les Inuit et les Blancs on mélange les pommes et les racines carrées. On se dit aussi que bien des ethnies d'Occident ont été les premières surprises d'apprendre qu'elles étaient d'Occident; que le chanoine Groulx (chantre du nationalisme de survivance canadien-français) et nos grand-parents, tout blancs qu'ils aient été, n'en ont pas moins souffert des oppressions qu'au pays des Inuit on attribue au Blanc ${ }^{27}$; qu'enfin, quelque part en Asie, il y a des gens pour qui les Blancs s'appellent des Japonais, ou qu'on trouve en Bretagne des Bretons qui ne sont pas loin de considérer les Français hexagonaux comme une espèce de Blancs.

Alors? Il est certain que dans le milieu Inuit, le Blanc représente autre chose qu'un super-groupe ethnique. Il représente le Capitalisme, le Colonisateur, l'État, la Bourgeoisie, la Technobureaucratie. Cet article au complet ne s'est pas occupé d'autre chose, vraiment, que de le dire et de l'illustrer.

Oui, mais. L'esprit critique et l'humanisme qui inspirent, dans la bouche même des Inuit (ou des Africains) l'analyse et la dénonciation du colonialisme culturel ou de l'ethnocentrisme occidentaux remontent eux-mêmes aux Grecs, à l'Europe des Lumières, à la scolarisation généralisée - donc, aux Blancs. Si le capitalisme est d'Occident, il faut bien admettre que le socialisme dont se réclament les Chinois ou les Angolais n'est pas issu non plus de Chine ou des cultures empaysées dans ce qui porte aujourd'hui le nom d'Angola. Se pourrait-il, en somme, qu'une mutation fondamentale du rapport des êtres humains au monde se soit produite, par les hasards de l'histoire, autour du quinzième siècle et aux environs du versant nord de la Méditerranée pour s'étendre sur la planète entière à partir de sa souche «blanche», accidentelle et contingente? Que le capitalisme, puisqu'il faut l'appeler par son nom, ait fait naître quelque chose qui le dépasse et qu'il ne saurait contenir éternellement? Plus qu'une culture parmi d'autres : un mode sans précédent d'appropriation symbolique et matérielle du monde, de portée universelle et - je n'ai pas dit: indépassable - irréversible?

Au lieu de répondre, je pense à l'histoire d'Abel et de Caïn. Le premier est un pâtre. Le second, agriculteur. Les fils de Caïn fonderont la ville; Tubalcaïn, de la même lignée, inventera la forge. Derrière le meurtre d'Abel, je vois la destruction d'une civilisation nomade de chasse, de cueillette, et de transhumance bergère par une civilisation sédentaire, agro-artisanale, urbaine, et, on le soupçonne, étatique, littéraire, de classe, théocratique. Je vois la révolution néolithique racontée par une tribu mésoorientale qui en fut victime, pour qui elle prit la forme du baton colonial.

L'œil était dans la tombe et regardait Caïn, dit la strophe d'Hugo. Il en va de même du fardeau de l'Homme Blanc. Avouons que d'aucuns font de l'analyse sociologique pour essayer de s'en décharger. Partiellement.

\section{RÉSUMÉ}

Le discours critique sur le colonialisme intérieur canadien dénonce couramment l'oppression qu'exerce le Blanc sur l'Autochtone. Prenant les Inuit pour cas d'espèce, on essaie de faire voir ce que dissimule cette simplification manichéiste. Que l'altérité du Blanc est une des composantes de l'identité inuite, et que ce rapport identitaire emprunte à un même champ de signification. Que la réification idéologique de l'Inuicité et du fardeau de l'Homme blanc répond à l'impératif d'expropriation des premiers habitants et justifie le ghetto technobureaucratique où ils se trouvent aujourd'hui enfermés, en marge de l'espace et de l'histoire.

\section{SUMMARY}

The critical discourse on Canadian internal colonialism frequently denounces the oppression of Natives by Whites. Taking the Inuit as a case in point, an attempt is made to demonstrate what hides behind such a gross oversimplification : 1) that the otherness of Whites is one of the elements making up the Inuit identity, and that this identity relationship borrows from one and the same field of meaning; 2) that the ideological reification of Inuit- 
ness and of the White man's burden is a response to the imperatives of expropriation of the first inhabitants and justifies the techno-bureaucratic ghetto into which they are locked today, on the fringes of space and of history.

\section{RESUMEN}

El discurso crítico sobre el colonialismo interno canadiense denuncia corrientemente la opresión que ejerce el Blanco sobre el Autóctono. Tomando a los Inuit como especie, se trata de hacer ver lo que disimula esta simplificación maniqueísta. Que la diferencia del Inuit con relación al Blanco es uno de los componentes de su propia identidad, y que esta relación de identidad pertenece a un mismo terreno de significación. Que la visión ideológica estática de la «Inuicidad» y del peso del hombre blanco responden al imperativo de expropiación de los primeros habitantes y justifica el ghetto tecno-burocrático en el cual se encuentran encerrados actualmente, al margen del espacio y de la historia. 